\title{
NOTE
}

\section{Carrier Mobility for $\pi$-Conjugated Dendrimer Composed of Phenylene-vinylene Dendron and Triphenylamine Surface Group}

\author{
By Masanobu MIZUSAKI, ${ }^{1, *}$ Motohiro YAMAHARA,,${ }^{1, \dagger}$ Yuichiro YAMADA, ${ }^{1}$ \\ Satoru OBARA, ${ }^{2}$ and Kentaro TADA ${ }^{2}$
}

KEY WORDS: $\pi$-Conjugated Soluble Dendrimer / Generation Number / Mobility / Time of Flight /

Carrier transport property of $\pi$-conjugated dendrimer is of current scientific and technological interest because of a molecular architecture for organic semiconductor materials and because of a variety of practical applications such as light emitting diodes, thin film transistors, and photo-voltaic cells. ${ }^{1-6}$

Previously, we reported the carrier transport property of the soluble $\pi$-conjugated three-generation dendrimer which is composed of phenylene-vinylene as a dendron group and triphenylamine as a soluble surface group (G3D3-PV(TPA)) as shown in Figure 1b. ${ }^{7}$ The carrier of transporting across the G3D3-PV(TPA) dendrimer film was preferentially hole with an indium-tin-oxide (ITO)/G3D3-PV(TPA)/Mg-Al cell. The Mg$\mathrm{Al}$ is the opposite electrode composed of alloy of $\mathrm{Mg}$ and $\mathrm{Al}$. The hole mobility was $10^{-5}\left(\mathrm{~cm}^{2} /(\mathrm{V} \cdot \mathrm{s})\right)$ order at $25^{\circ} \mathrm{C}$, which is the same order as that of poly( $p$-phenylene-vinylene) reported by Lebedev and co-workers though the dendrimer is surrounded by the triphenylamine surface group. ${ }^{8}$ According to the results reported previously, the triphenylamine surface group covalently attached to the dendron did not show the carrier transport property. ${ }^{7}$ This result indicates that the phenylene-vinylene molecules with different dendrimer are comparably close to each other. We have estimated the structure of the G3D3-PV(TPA) dendrimer was comparably planar shape rather than globular shape. ${ }^{7}$

If the above conclusion is not contradicted, the dendrimer of the lower generation number is expected to become higher mobility because the dendron and surface groups are less restricted by each moiety, and the dendrimer would be more planar shape. To examine the effect of steric constraints by decrement of the generation number on the carrier mobility, we prepared a soluble $\pi$-conjugated two-generation dendrimer G2D3-PV(TPA) as shown in Figure 1a, where the phenylenevinylene dendron and the triphenylamine surface group are less restricted. In this dendrimer, the structure is expected to be more planar under less constraining condition than in the case of the G3D3-PV(TPA) dendrimer.
In this paper, we have reported the carrier transport property of the G2D3-PV(TPA) dendrimer with the ITO/G2D3$\mathrm{PV}(\mathrm{TPA}) / \mathrm{Mg}-\mathrm{Al}$ cell by a time-of-flight (TOF) method focusing on the comparison of the three-generation dendrimer.

\section{EXPERIMENTAL}

The chemical structure of the two-generation dendrimer (G2D3-PV(TPA)) used in this study is shown in Figure 1a. The synthesis procedure was reported previously. ${ }^{9}$ The weight and number average molecular weights measured by GPC were 4,500 and 4,300, respectively, and the molecular weight distribution was 1.05. ${ }^{9}$ The standard polymer and eluent for GPC evaluation were polystylene and THF, respectively. For comparison, we also used the three-generation dendrimer G3D3-PV(TPA) shown in Figure 1b.

The solutions of the dendrimers were prepared in chloroform at concentrations of $10 \mathrm{mg} / \mathrm{mL}$. The ITO substrates were masked, patterned and cleaned in ultrasonic of $\mathrm{NaOH}$ and deionized water. Films of the dendrimers, typically $1 \mu \mathrm{m}$ thick, were formed by spin-coating from the chloroform solutions onto the cleaned ITO substrates at $25^{\circ} \mathrm{C}$. A vacuum evaporator was used for the thermal evaporation of the alloy of $10 \mathrm{wt} \%$ $\mathrm{Mg}$ and $90 \mathrm{wt} \% \mathrm{Al}$ (Mg-Al) onto the desired ITO glass plates deposited with the $1 \mu \mathrm{m}$ dendrimer films.

TOF measurements for the ITO/dendrimer/Mg-Al cells were carried out with a conventional setup as described previously. ${ }^{7}$ The measurements were performed at $25^{\circ} \mathrm{C}$ in vacuum condition $\left(10^{-3}\right.$ Torr $)$. The current generated as a result of photocarrier drift was collected across a $50 \Omega$ resister upon the application of an external bias voltage for $100 \mathrm{~ms}$. The carrier mobility was determined by the following relation;

$$
\mu=\frac{L^{2}}{T_{\text {tr }} V}
$$

where $\mu$ is the carrier mobility, $L$ is the thickness of the

\footnotetext{
${ }^{1}$ Corporate Research and Development Group, Sharp Corp., 2613-1 Ichinomoto-cho, Tenri 632-8567, Japan

${ }^{2}$ Photosensitive Materials Research Center, Toyo Gosei Co. Ltd., Wakahagi, 4-2-1 Inba-mura, Inba-gun 270-1609, Japan

'Present Address: SUMITOMO CHEMICAL Co. Ltd. IT-Related Chemicals Research Laboratory

*To whom correspondence should be addressed (Tel: +81-743-65-3876, Fax: +81-743-65-4045, E-mail: mizusaki.masanobu@ sharp.co.jp).
} 
(a) G2D3-PV(TPA)

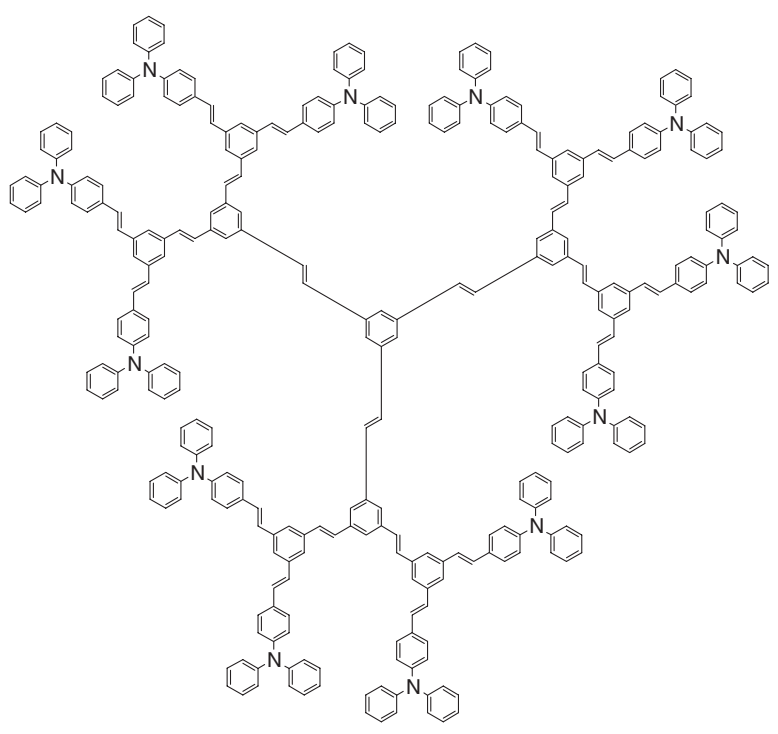

(b) G3D3-PV(TPA)

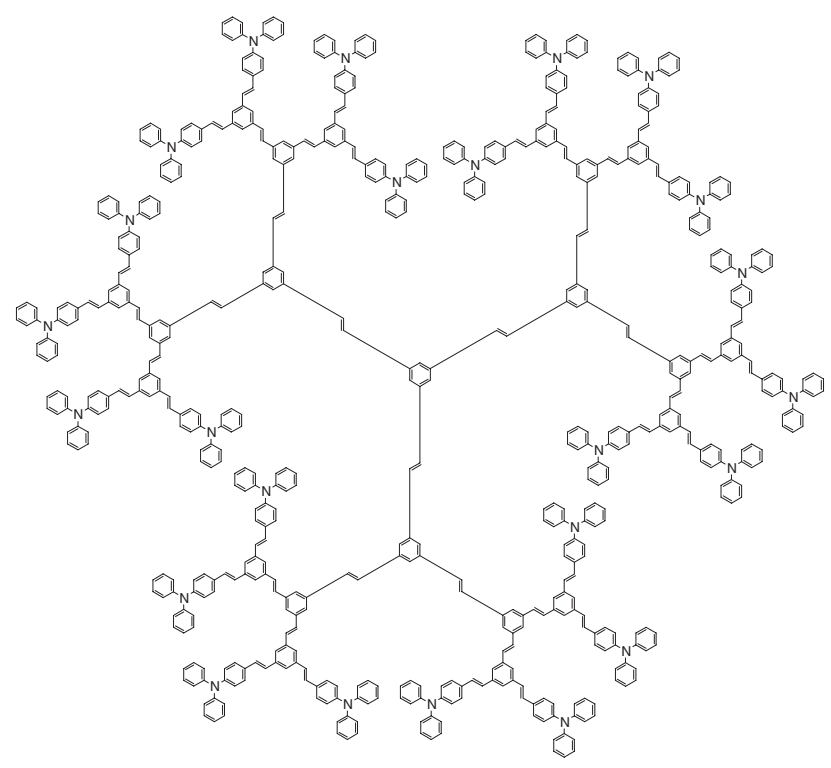

Figure 1. (a) G2D3-PV(TPA) dendrimer used in this study and (b) G3D3$\mathrm{PV}(\mathrm{TPA})$ dendrimer for comparison as a reference in this study.

dendrimer film, $V$ is the applied bias voltage, and $T_{\text {tr }}$ is the transit time derived from the double logarithmic plot of the transient photocurrent. ${ }^{7}$

\section{RESULTS AND DISCUSSION}

By illuminating a pulsed $\mathrm{N}_{2}$ laser $(337.1 \mathrm{~nm}$, pulse width $0.7 \mathrm{~ns}$ ) from the ITO side of the ITO/dendrimer/Mg-Al cell, photoexcited carriers of hole and electron generate at the surface of the dendrimer film since the absorption of the dendrimer occurs at $337.1 \mathrm{~nm}$ shown in Figure 2. As we reported previously, the absorption centered at $370 \mathrm{~nm}$ is from the phenylene-vinylene site and that centered at $300 \mathrm{~nm}$ is from

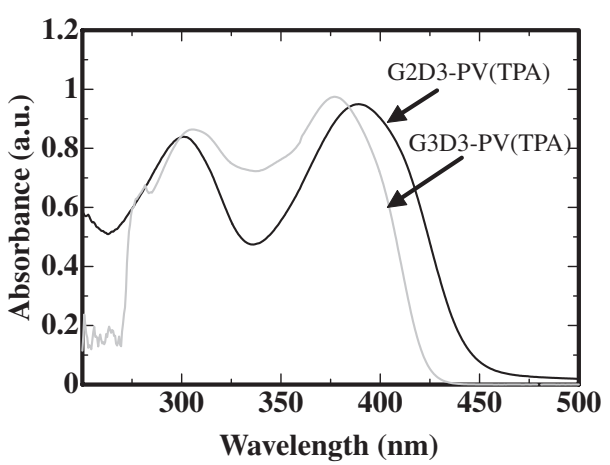

Figure 2. UV absorption spectra for G2D3-PV(TPA) and G3D3-PV(TPA).

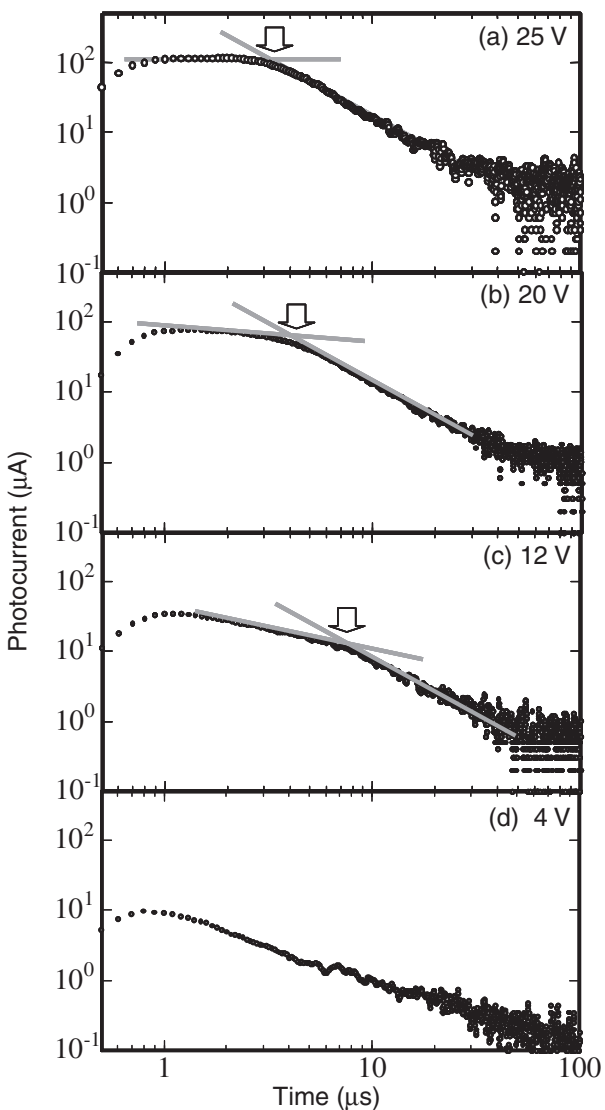

Figure 3. Profiles of double logarithmic transient photocurrent plots for ITO/ G2P3-PV(TPA)/Mg-Al device at applied voltage of (a) $25 \mathrm{~V}$ (b) $20 \mathrm{~V}$, (c) $12 \mathrm{~V}$, and (d) $4 \mathrm{~V}$.

the triphenylamine group. ${ }^{7}$ In the case that the positive bias voltage was applied at the ITO side, transient photocurrent was observed, indicating the hole drift occurred from the ITO side to the $\mathrm{Mg}-\mathrm{Al}$ side. On the other hand, when the negative bias voltage was applied at the ITO side, transient photocurrent was not observed, implying the electron did not drift. The double logarithmic plots of the transient photocurrents of the hole for the ITO/G2D3-PV(TPA)/Mg-Al cell in the $V$ range from $25 \mathrm{~V}$ to $4 \mathrm{~V}$ are shown in Figure $3 \mathrm{a}-3 \mathrm{~d}$. $T_{\text {tr }}$, which is determined from the intercept of two tangential lines before and after the inflection point of the photocurrent, is clearly observed at 


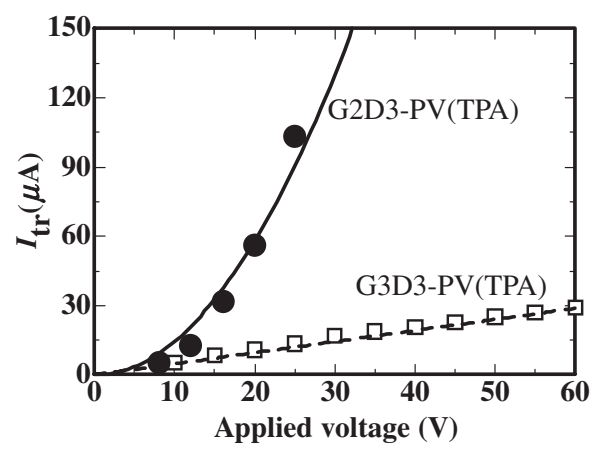

Figure 4. Current at the transit time $I_{\text {tr }}$ for G2D3-PV(TPA) and G3D3PV(TPA) as a function of $V$.

comparably high $V$ whereas it becomes difficult to distinguish $T_{\text {tr }}$ with decreasing $V$ as shown in Figure $3 \mathrm{~d}$. The fact that the inflection point becomes unobservable with decreasing $V$ implies that the hole disappears before arriving at the $\mathrm{Mg}-\mathrm{Al}$ side. One of the reasons for the disappearance is that the recombination occurs preferentially between the hole and the electron at the surface of the dendrimer film at low $V$ since the transport velocity of the hole is small. On the other hand, as $V$ becomes high, the transport velocity becomes high and the rate of the recombination becomes law.

Figure 4 shows the current value at the inflection point $\left(I_{\text {tr }}\right)$ for G2D3-PV(TPA) as a function of $V$. For comparison, $I_{\mathrm{tr}}$ for G3D3-PV(TPA) is also shown in this figure. The $I_{\text {tr }}$ values for G2D3-PV(TPA) are much higher than those for G3D3PV(TPA). There are two reasons of this. One is that the mobility for G2D3-PV(TPA) is higher than that for G3D3PV(TPA), the other is that the number of the carrier drifting across the dendrimer film for G2D3-PV(TPA) is higher than that for G3D3-PV(TPA). The result leads to the fact that the steric structure of G2D3-PV(TPA) is strikingly different from that of G3D3-PV(TPA) owing to the less restricted steric barrier. This implies the possibility that the mobility or the number of the carrier drifting across the film increases nonlinearly with increasing $V$ for G2D3-PV(TPA).

The hole mobility, which was determined with eq 1 , for G2D3-PV(TPA) and G3D3-PV(TPA) as a function of the square root of the applied bias voltage $V^{1 / 2}$ are depicted in Figure 5. The mobility for G2D3-PV(TPA) is about three times higher than that for G3D3-PV(TPA) in the $V^{1 / 2}$ range from $2.8 V^{1 / 2}$ to $5 V^{1 / 2}$. The higher mobility for G2D3-PV(TPA) is induced by the easier carrier hopping compared with the case of G3D3-PV(TPA). We estimate that the distance between the phenylene-vinylene dendrons with the different dendrimer molecule for G2D3-PV(TPA) is more close to each other than that for G3D3-PV(TPA). As those dendrimers are surrounded by the triphenylamine surface group, the dendrimers need to be planar structure for the dendron site, which is the carrier transporting site, to become close to each other and show high mobility. According to the comparisons for $I_{\text {tr }}$ and hole mobility between G2D3-PV(TPA) and G3D3-PV(TPA), the degree of the planar structure for G2D3-PV(TPA) is estimated

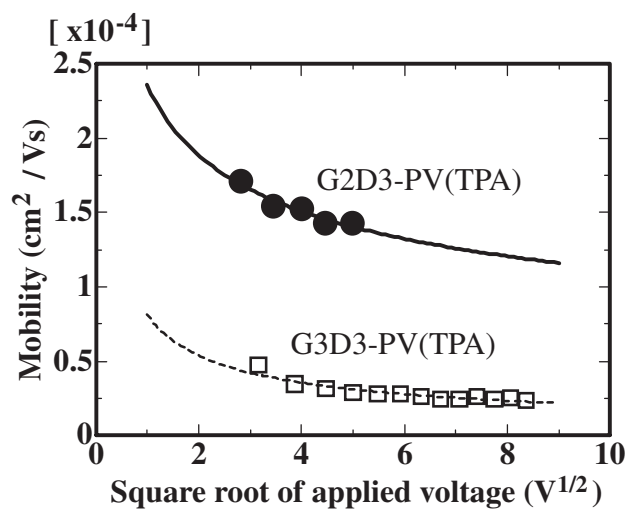

Figure 5. Hole Mobility for G2D3-PV(TPA) and G3D3-PV(TPA) as a function of $V^{1 / 2}$.

to be higher than that for G3D3-PV(TPA). Here, the mobility for both the dendrimers decreased with increasing the application voltage. Concerning this, Bassler proposed a model to explain the various electric field dependence of the mobility by the Monte Carlo simulation of random carrier walk. ${ }^{10}$ Based on the model, in the case of low bias voltage application, some of the carriers transport to the opposite electric field direction, resulting in the negative electric field dependence of the mobility.

We succeeded to improve the carrier mobility for the $\pi$ conjugated dendrimer having soluble surface group to $10^{-4}$ $\left(\mathrm{cm}^{2} /(\mathrm{V} \cdot \mathrm{s})\right)$ order by decreasing the generation number of the dendron site. This is owing to the facts that less restricted structure is formed and that the distance between the $\pi$ conjugated dendron sites with the different dendrimer is more close to each other.

Received: January 9, 2009

Accepted: March 5, 2009

Published: April 15, 2009

\section{REFERENCES}

1. J. P. J. Markham, I. D. W. Samuel, S.-C. Lo, P. L. Burn, M. Weiter, and H. Bassler, J. Appl. Phys., 95, 438 (2004).

2. J. M. Lupton, I. D. W. Samuel, R. Beavington, M. J. Frampton, P. L. Burn, and H. Bassler, Phys. Rev. B: Condens. Matter Mater. Phys., 63, 155206 (2001).

3. J. M. Lupton, I. D. W. Samuel, R. Beavington, P. L. Burn, and H. Bassler, Synth. Met., 116, 357 (2001).

4. J. M. Lupton, I. D. W. Samuel, R. Beavington, P. L. Burn, and H. Bassler, Adv. Mater., 13, 258 (2001).

5. T. D. Anthopoulos, J. P. J. Markham, E. B. Namdas, J. R. Lawrence, I. D. W. Samuel, S.-C. Lo, and P. L. Burn, Org. Electron., 4, 71 (2003).

6. S. Gambino, I. D. W. Samuel, H. Barcena, and P. L. Burn, Org. Electron., 9, 220 (2008).

7. M. Mizusaki, M. Yamahara, Y. Yamada, S. Obara, and K. Tada, Polym. J., 41, 195 (2009).

8. E. Lebedev, Th. Dittrich, V. Petrova-Koch, S. Karg, and W. Brutting, Appl. Phys. Lett., 71, 2686 (1997).

9. M. Yamahara, S. Obara, and K. Tada, Jpn Patent No. 2004-18539 (2004).

10. H. Bassler, Phys. Status Solidi B, 175, 15 (1993). 